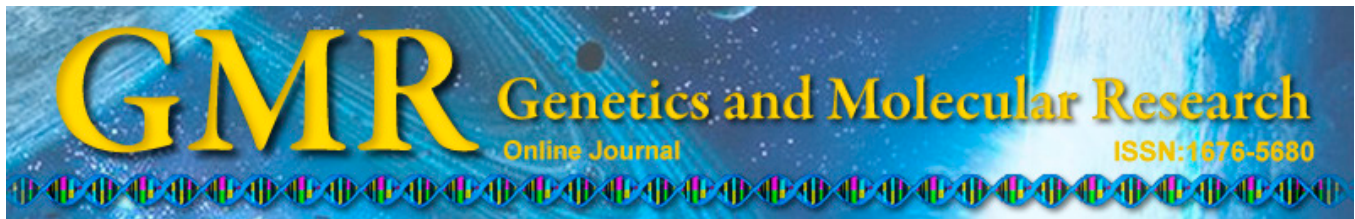

\title{
Relation between prognosis and changes of MBP and S100B in premature infants with periventricular leukomalacia
}

R.Z. Huang, Y.J. Zhang, J.F. Zhang, Y.M. Su, L.Q. Peng and N. Ya

Department of Pediatrics, Maternal and Child Healthcare of Shunde District, Foshan, Guangdong Province, China

Corresponding author: R.Z. Huang

E-mail: RunzhongHuang@126.com

Genet. Mol. Res. 14 (2): 4338-4343 (2015)

Received May 14, 2014

Accepted October 30, 2014

Published April 30, 2015

DOI http://dx.doi.org/10.4238/2015.April.30.6

\begin{abstract}
This study aims to explore the relation between changes in myelin basic protein (MBP) and S100 protein (S100B) serum levels and prognosis in premature infants with periventricular leukomalacia (PVL). In our hospital, 78 premature infants with PVL and 43 normal premature infants were studied from July 1, 2007 to December 31, 2008. MBP and S100B serum levels were detected at 1, 3, 7, and 14 days after birth by using enzyme-linked immunosorbent assay. All infants were followed four times (once every 3 months) after discharge from hospital. Their intelligence quotient and physical development index were tested by using Gesell developmental scales. The MBP serum levels were significantly higher in premature infants with PVL at any time point than in normal premature infants. S100B serum levels gradually increased at 1, 3, and 7 days; peaked on the 7th day; and then gradually decreased to the normal level on the 14th day. The intelligence quatient and physical development index of infants with increased S100B and MBP levels on the 7th day were lower than those of infants who had normal S100B and MBP levels and those of normal premature infants. A negative relation exists between S100B and MBP
\end{abstract}


serum levels and prognosis in PVL infants. An increase of MBP and S100B levels lasting $>7$ days could cause poor prognosis.

Key words: Premature infants; Myelin basic protein; S100B; Periventricular leukomalacia

\section{INTRODUCTION}

Periventricular leukomalacia (PVL) in premature infants leads to the development of cerebral palsy and dysnoesia (Volpe, 2009; Lee et al., 2011; Kouwaki et al., 2013). Myelin basic protein (MBP) is distributed in central and peripheral nerves. S100 protein (S100B) is localized in neuroglial cells. When neurocytes and neuroglial cells undergo necrosis, MBP and S100B are leaked and pass through the injured blood-brain barrier, resulting in an increase in their concentration in serum (Subspecialty Group of Neonatology, Society of Pediatrics, Chinese Medical Association and the Editorial Board of Chinese Journal of Pediatrics, 2007; Chen et al., 2008; Hernandez-Cabrera et al., 2009). Our study investigated the relation between prognosis and the changes in S100B and MBP levels in premature infants with PVL, through a follow-up of the infants and monitoring of the serum concentration of MBP and S100B.

\section{MATERIAL AND METHODS}

\section{Data and group}

In our hospital, all premature infants were evaluated from November 1, 2007 to July 1,2008 , including 75 male and 46 female infants with a gestational age of $<34$ weeks. All infants had cranial B-ultrasound on the 3rd, 4th, and 7th days after birth. Moreover, cranial magnetic resonance imaging (MRI) was performed when the premature infants were in a stable condition. According to the results of cranial B-ultrasonography and MRI, all preterm infants were divided into two groups: the normal group (43 infants with normal results) and the PVL group (78 infants with PVL; mild PVL in 58 infants and moderate PVL in 20 infants). There was no significant difference between gestational age, weight, Apgar scores, and other parameters.

\section{PVL diagnosis and grading standards}

Previously published references were consulted concerning the diagnostic suggestions for periventricular-intraventricular hemorrhage and PVL in premature infants and the grading criteria for PVL (Deng et al., 2008).

\section{Index detection}

Blood samples were collected from premature infants, which were then centrifuged at $3000 \mathrm{rpm}$ to obtain the supernatant fluid and stored at $-40^{\circ} \mathrm{C}$. All samples were tested by using the enzyme-linked immunosorbent assay (ELISA). The ELISA reagent kit was purchased from BIOWEN Company (Canada). 


\section{Treatment}

Infants from the two groups were treated by using a routine anti-infection regimen to maintain homoeostatic parameters such as temperature, blood pressure, blood glucose, and water-electrolyte level.

\section{Follow-up}

All infants were followed once a month until they were 1 year old, and Gesell developmental scales were used to test the intelligence quotient and physical development index of the premature infants once every 3 months (Paintlia et al., 2008; Haynes and van Leyen, 2013).

\section{Statistical analysis}

Statistical analysis was performed by using the SPSS 12.0 software, and all results are reported as means $\pm \mathrm{SE}$. The least significant difference $t$-test was used for two-sample comparisons between the two groups. One-way analysis of variance was used to compare differences between groups. A P value of $<0.05$ was considered to denote a significant statistical difference.

\section{RESULTS}

\section{Change of S100B levels}

The S100B levels of PVL infants were significantly increased at 1, 3, and 7 days, and were highest on the 7th day. There was a significant difference between the normal group and the PVL group. As the infants' condition improved, the S100B serum level decreased in the PVL group. There was no significant difference between the two groups on the 14th day (Table 1).

Table 1. Changes in S100B serum levels on the 1st, 3rd, 7th, and 14th days in the two groups $(\mu \mathrm{g} / \mathrm{L})$.

\begin{tabular}{lcccccc}
\hline & $\mathrm{N}$ & 1st day & 3rd day & 7th day & 14th day & F value \\
\hline NG & 43 & $2.46 \pm 0.77$ & $2.61 \pm 0.91$ & $2.93 \pm 1.20$ & $2.38 \pm 0.87$ & 1.28 \\
PVLG & 78 & $3.82 \pm 0.68$ & $4.41 \pm 0.91$ & $5.78 \pm 1.54$ & $3.27 \pm 0.86$ & 5.36 \\
$t$ value & & 2.34 & 3.76 & 4.22 & 1.87 & $<0.05$ \\
P value & & $<0.05$ & $<0.01$ & $<0.001$ & $>0.05$ & \\
\hline
\end{tabular}

NG, normal group; PVLG, periventricular leukomalacia group.

\section{Change of MBP serum levels}

The MBP serum levels reached maximum on the 3rd day in the PVL group. With the improvement of the infants' condition, the MBP levels gradually decreased, and there were significant differences at different time points. In addition, serum MBP levels were much higher at 1,3, 7, and 14 days after birth in the PVL group than in the normal group (Table 2). 
Table 2. Changes in serum MBP levels on the 1st, 3rd, 7th, and 14th days in the two groups $(\mu \mathrm{g} / \mathrm{L})$.

\begin{tabular}{|c|c|c|c|c|c|c|c|}
\hline & $\mathrm{N}$ & 1st day & 3rd day & 7th day & 14th day & F value & $P$ value \\
\hline $\mathrm{NG}$ & 43 & $5.41 \pm 0.51$ & $5.42 \pm 0.52$ & $5.43 \pm 0.50$ & $5.47 \pm 0.50$ & 0.68 & $>0.05$ \\
\hline PVLG & 78 & $7.61 \pm 1.78$ & $14.53 \pm 3.12$ & $10.86 \pm 1.54$ & $7.89 \pm 1.77$ & 6.23 & $<0.05$ \\
\hline$t$ value & & 2.07 & 5.64 & 3.86 & 2.32 & & \\
\hline$P$ value & & $<0.05$ & $<0.001$ & $<0.001$ & $<0.05$ & & \\
\hline
\end{tabular}

NG, normal group; PVLG, periventricular leukomalacia group.

\section{Intelligence and physical development quotient test}

According to the concentration of MBP $(>10.0 \mu \mathrm{g} / \mathrm{L})$ and S100B $(>4.5 \mu \mathrm{g} / \mathrm{L})$ on the 7th day after birth, premature infants were divided into three groups: normal group (group 1), group with an obvious decrease of S100B and MBP (group 2), and group with a continuous increase of MBP and S100B (group 3). We found that the development quotient scores were significantly lower in group 3 than those in groups 1 and 2 (Table 3).

Table 3. Relation between development quotient and protein levels of S100B and MBP on the 7th day after birth.

\begin{tabular}{lcccr}
\hline Group & $\mathrm{N}$ & $\mathrm{S} 100 \mathrm{~B}$ & $\mathrm{MBP}$ & Development quotient \\
\hline Group 1 & 30 & $2.93 \pm 1.20$ & $5.43 \pm 0.50$ & $101.07 \pm 7.67$ \\
Group 2 & 42 & $3.82 \pm 1.02$ & $5.60 \pm 0.92$ & $98.07 \pm 6.68$ \\
Group 3 & 27 & $6.08 \pm 1.94$ & $13.48 \pm 1.94$ & $83.43 \pm 7.88$ \\
F value & & 6.67 & $<.05$ & 3.58 \\
P value & & $<0.05$ & $<0.01$ & $<0.05$ \\
\hline
\end{tabular}

\section{DISCUSSION}

PVL is a major causative factor of cerebral palsy in premature infants. Defective development of brain tissues and relatively less vascularity around the alba lead to cerebral white damage in premature infants, especially in those with gestational ages $<34$ weeks (De Vries et al., 2004; Steiner et al., 2007; Kleindienst et al., 2010). The rate of incidence of PVL is between 2.3 and 7.5\% (Beers et al., 2007; Berger et al., 2007).

Concerning the prognosis of PVL infants, many studies have reported that on ultrasound and cranial MRI detection, the presence of more than three cysts in cerebral white matter, multicyst formation, and PVL are predictors of poor prognosis (Douglas-Escobar and Weiss, 2013); however, few studies have focused on the early prediction of prognosis in newborns.

S100B is mainly distributed in neuroglial and Schwann cells of the central and peripheral nervous systems (Subspecialty Group of Neonatology, Society of Pediatrics, Chinese Medical Association and the Editorial Board of Chinese Journal of Pediatrics, 2007). S100B cannot penetrate the blood-brain barrier in the normal condition; however, when neuroglial cells undergo necrosis, $\mathrm{S} 100 \mathrm{~B}$ is leaked and penetrates across the injured blood-brain barrier, increasing its level in serum (Chen et al., 2008). The serum S100B level reflects the degree of damage, and is useful in the evaluation of the therapeutic efficiency of drugs and of the prognosis of the central nervous system (Fishler et al., 1965; Egberts et al., 2008; Murabayashi et al., 2008; Berger et al., 2010; Gyorgy et al., 2011). In this study, the results showed that the S100B level of preterm infants with cerebral white damage 1 day after birth had no significant difference with that of normal preterm infants, and that S100B concentration increased on the 
3rd day and peaked on the 7th day; however, with the improvement of body condition, there was an obvious decrease on the 14th day. Moreover, infants with worse condition showed higher serum S100B concentrations. Murabayashi et al. (2008) had a similar report to the Subspecialty Group of Neonatology, Society of Pediatrics, Chinese Medical Association and the Editorial Board of Chinese Journal of Pediatrics (2007). The development quotients of premature infants with PVL, whose S100B serum levels were still increased on the 7th day, were significantly lower than those of normal premature infants, which indicates that premature infants with PVL had a poor prognosis. The S100B concentrations of two infants were $>10$ $\mu \mathrm{g} / \mathrm{L}$, and follow-up of these two infants showed that they have cerebral palsy.

MBP is a major protein believed to be important in the myelination of nerves in the nervous system. Hypoxia-ischemia damages neurocytes and neuroglial cells, causing MBP to enter into the bloodstream (Egberts et al., 2008; Murabayashi et al., 2008; Gyorgy et al., 2011). Therefore, serum MBP could reflect brain injury severity, therapeutic efficiency, and prognosis (Egberts et al., 2008; Murabayashi et al., 2008; Gyorgy et al., 2011; Zhou et al., 2013). Berger et al. (2010) reported that the detection of S100B and MBP serum concentrations is important for predicting prognosis, and that MBP had an accuracy of $73 \%$ and specificity of $61 \%$, and S100B had a sensitivity of 59\% and specificity of 100\%. Gyorgy et al. (2011) also demonstrated that higher S100B and MBP serum levels indicated poorer prognosis. Our results showed that the MBP serum level of preterm infants with cerebral white damage, at 1 day after birth, was not significantly different from that of normal preterm infants. Moreover, MBP concentration increased on the 3rd day and peaked on the 7th day; however, with the improvement of the infants' condition, there was an obvious decrease on the 14th day. Furthermore, the follow-up found that the development quotients of premature infants with PVL, whose MBP serum levels were still increased on the 7th day, were significantly lower than those of normal premature infants, which indicates that severe brain injury in premature infants with PVL could increase the incidence rate of sequelae.

In conclusion, the dynamic changes of S100B and MBP serum levels could predict prognosis. Continual increase of S100B and MBP serum levels could indicate severe brain injury and poor prognosis, and cranial ultrasound and MRI can enhance the accuracy of results. However, our studies included only a limited number of cases, and a multicenter large-scale study is needed in the future.

\section{REFERENCES}

Beers SR, Berger RP and Adelson PD (2007). Neurocognitive outcome and serum biomarkers in inflicted versus noninflicted traumatic brain injury in young children. J. Neurotrauma 24: 97-105.

Berger RP, Beers SR, Richichi R, Wiesman D, et al. (2007). Serum biomarker concentrations and outcome after pediatric traumatic brain injury. J. Neurotrauma 24: 1793-1801.

Berger RP, Bazaco MC, Wagner AK, Kochanek PM, et al. (2010). Trajectory analysis of serum biomarker concentration facilitates outcome prediction after pediatric traumatic and hypoxemic brain injury. Dev. Neurosci. 32: 396-405.

Chen HJ, Wei KL and Yao YJ (2008). Multicenter investigation for incidence of periventricular leukomalacia in premature infants in China. Zhongguo Dang Dai Er Ke Za Zhi 10: 686-692.

De Vries LS, Van Haastert IL, Rademaker KJ, Koopman C, et al. (2004). Ultrasound abnormalities preceding cerebral palsy in high-risk preterm infants. J. Pediatr. 144: 815-820.

Deng W, Pleasure J and Pleasure D (2008). Progress in periventricular leukomalacia. Arch. Neurol. 65: 1291-1295.

Douglas-Escobar M and Weiss MD (2013). Biomarkers of brain injury in the premature infant. Front. Neurol. 3: 185.

Egberts F, Pollex A, Egberts JH, Kaehler KC, et al. (2008). Long-term survival analysis in metastatic melanoma: serum S100B is an independent prognostic marker and superior to LDH. Onkologie 31: 380-384.

Fishler K, Graliker BV and Koch R (1965). The predictability of intelligence Gesell developmental scales in mentally 
retarded infants and young children. Am. J. Ment. Defic. 69: 515-525.

Gyorgy A, Ling G, Wingo D, Walker J, et al. (2011). Time-dependent changes in serum biomarker levels after blast traumatic injury. J. Neurotrauma 28: 1121-1126.

Haynes RL and van Leyen K (2013). 12/15-Lipoxygenase expression is increased in oligodendrocytes and microglia of periventricular leukomalacia. Dev. Neurol. 35: 140-154.

Hernandez-Cabrera MA, Flores-Santos R, Garcia-Quintanilla JF, Hernández-Herrera RJ, et al. (2009). Periventricular leukomalacia prevalence in premature newborn. Rev. Med. Inst. Mex. Seguro Soc. 47: 147-150.

Kleindienst A, Melssner S, Eyupoglu IY, Parsch H, et al (2010). Dynamics of S100B release into serum and cerebrospinal fluid following acute brain injury. Acta Neurochir. Suppl. 106: 247-250.

Kouwaki M, Yokochi M, Togawa Y, Kamiya T, et al. (2013). Spontaneous movements in the supine position of healthy term infants and preterm infants with or without periventricular leukomalacia. Brain Dev. 35: 340-348.

Lee JD, Park HJ, Park ES, Oh MK, et al. (2011). Motor pathway injury in patients with periventricular leukomalacia and spastic diplegia. Brain 134: 199-210.

Murabayashi M, Minato M, Okuhata Y, Makimoto M, et al. (2008). Kinetics of serum S100B in newborns with intracranial lesions. Pediatr. Int. 50: 17-22.

Paintlia MK, Paintlia AS, Contreras MA, Singh I, et al. (2008). Lipopolysaccharide-induced peroxisomal dysfunction exacerbates cerebral white matter injury: attenuation by $N$-acetyl cysteine. Exp. Neurol. 210: 560-576.

Steiner J, Bermstein HG and Blelau H (2007). Evidence for a wide extra-astrocytic distribution of S100B in human brain. BMC Neurosci. 8: 2.

Subspecialty Group of Neonatology, Society of Pediatrics, Chinese Medical Association and the Editorial Board of Chinese Journal of Pediatrics (2007). Diagnostic suggestions for periventricular-intraventricular hemorrhage and periventricular leukomalacia in premature infants. Zhonghua Er Ke Za Zhi 45: 34-36.

Volpe JJ (2009). Brain injury in premature infants: a complex amalgam of destructive and developmental disturbances. Lancet Neurol. 8: 110-124.

Zhou WJ, Liang AM, Wang FZ, et al. (2013). Epidemiological study on developmental delay of 18-month-old children from four districts/counties in Beijing. Beijing Da Xue Xue Bao 45: 211-216. 